\title{
Classification of Brain Tumors Using Hybridized Convolutional Neural Network in Brain MRI images
}

\author{
${ }^{1}$ Shwetha V, ${ }^{2} \mathrm{C}$ H Renu Madhavi, ${ }^{3}$ Nagendra Kumar M. \\ ${ }^{1}$ Department of Electronics \& Communication Engg, R V College of Engineering Bengaluru, \\ Affiliated to VTU Belagavi, also in Dept of ECE, S.J.C.Institute of Technology, Chickballapur, India \\ Email: shwethamtech@gmail.com
}

${ }^{2}$ Department of Electronics and Instrumentation Engineering, R V College of Engineering,Bengaluru,India.

Email: renumadhavi@rvce.edu.in

${ }^{3}$ Department of Electronics and Communication Engineering, S.J.C.Institute of

Technology,Chickballapur

Email: mnagendrakumar@sjcit.ac.in

Received: July 22, 2021. Revised: December 26, 2021. Accepted: January 13, 2022. Published: January 14, 2022.

\begin{abstract}
In this research article, we have proposed a novel technique to operate on the Magnetic Resonance Imaging (MRI) data images which can be classified as image classification, segmentation and image denoising. With the efficient utilization of MRI images the medical experts are able to identify the medical disorders such as tumors which are correspondent to the brain. The prime agenda of the study is to organize brain into healthy and brain with tumor in brain with the test MRI data as considered. The MRI based technique is an methodology to study brain tumor based information for the better detailing of the internal body images when compared to other technique such as Computed Tomography (CT).Initially the MRI image is denoised using Anisotropic diffusion filter, then MRI image is segmented using Morphological operations, to classify the images for the disorder CNN based hybrid technique is incorporated, which is associated with five different set of layers with the pairing of pooling and convolution layers for the comparatively improved performance than other existing technique. The considered data base for the designed model is a publicly available and tested KAGGLE database for the brain MRI images which has resulted in the accuracy of $88.1 \%$.
\end{abstract}

Keywords: Brain Tumour, CNN, Kaggle database, MRI.

\section{INTRODUCTION}

The most sensitive organ of our body is brain.It controls the core functions and characteristics in the human body.According to the National Brain Tumor Society, in the United States, about 7 lakh people live with a brain tumor, and the figure will rise to 7.87 lakh by the end of 2021 [1].

Compared to other cancers such as breast cancer or lung cancer, a brain tumor is uncommon but, still, a brain tumor is the number 10th leading cause of deaths worldwide. A tumor in brain has a lasting and psychological impact on patient life. The Brain tumor is caused by tissue abnormality that develops within the brain or in the central spine, interrupting proper brain function. The Brain tumor is diagnosed using several techniques such as CT scan, EEG and Magnetic Resource Image (MRI) [8].The representation of healthy brain and brain with tumor disorder with an MRI image is as depicted in the Fig. 1.

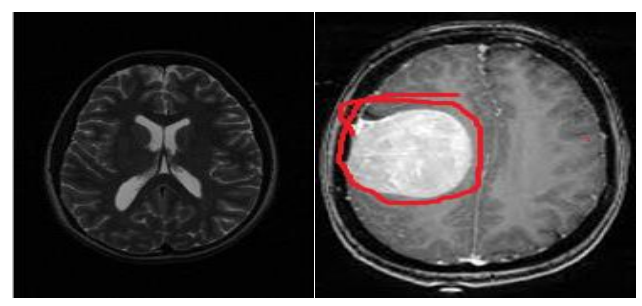

Figure 1. Healthy brain and Brain with Tumor [8]

The major improvement of the MRI based imaging when compared with the CT scan technique is the capturing of the all possible information in the test sample under consideration with minimized effect of the radiation on the medical subject under investigation.

While capturing the image with MRI technique a specific dye will be utilized for the classification among brain tumor cells and healthy brain cells under investigation. In conventional approach of brain image analysis which can be named as white matter, cerebrospinal liquid and grey matter, but for detailing analysis which are done using the three dimensional planar analysis such as axial, coronal and sagittal planes respectively as depicted in Fig. 2. The axial planar based analysis of images are captured from head to 
chin as shown in Fig. 2 (c), sagittal planar images are utilized for the right to left ear as represented in Fig. 2 (b) and with respect to Fig. 2 (a) coronal image based analysis. Similarly the few weights are considered individually for the analysis such as T1, T2 and Proton density weights for MRI analysis.

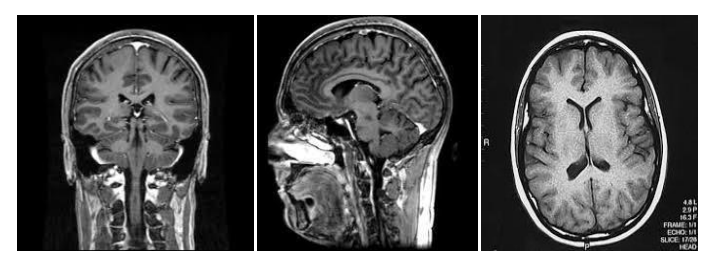

Figure 2. (a) Coronal image; (b) Sagittal image; (c) Axial image [5]

\section{REVIEW OF LITERATURE.}

In image processing techniques, primarily for tumour region spotting in image identification with segmentation it is deployed with the deep learning and AI (Artificial Intelligence). Till date an huge amount of works are carried in the domain of biomedical engineering.

Badza et al. [2] presented a new CNN architecture for brain tumor classification of three tumor types. The newly developed network is simpler than already-existing pretrained networks, and it was tested on T1-weighted contrastenhanced magnetic resonance images.Accuracy of $96.56 \%$ was achieved by them.

For both MRI and CT images,Ruba et al.[3] proposed a modified semantic segmentation networks (CNNs) based method The results showed that the proposed segmentation and classification yields overall accuracy of $99.57 \%, 99.78 \%$ and $99.56 \%$ for meningioma, glioma and pituitary tumors respectively.

Kalaiselvi et al.[4] used Recurrent Neural Network to classify the extracted features from brain. Experimental results showed that the proposed RNN classifier classifies the tumors in brain effectively with an accuracy of $98 \%$.

Cinarer et al.[5] proposed the Deep Neural Network classification model along with Synthetic Minority Oversampling Technique for preprocessing on Rembrandt Images dataset.The proposed method has achieved 95.0\% accuracy rate.Precision, Recall, F1-measure values are $95.4 \%, 95.0 \%$ and $94.9 \%$ respectively.

Sajja et al.[6] proposed hybridized CNN.Images from open databases such as BRATS were tested on brain MRI images.Accuracy of $96.15 \%$ was achieved using proposed model.

Khan et al.[7] presented an automated multimodal classification method using deep learning for brain tumor type classification. An accuracy of $97.8 \%, 96.9 \%, 92.5 \%$ for BraTs2015, BraTs2017, and BraTs2018, respectively, was achieved using proposed method.
Khan et al.[8] introduced the convolutional neural network $(\mathrm{CNN})$ approach along with Image Processing and Data Augmentation to categorize MRI scan images of brain into cancerous and non-cancerous. Experimental results showed that the proposed model is very effective and have very low complexity rate by achieving 100\% accuracy, while VGG-16 achieved 96\%, ResNet-50 achieved 89\% and Inception-V3 achieved 75\% accuracy.

Suganthe et al.[9] proposed a Recurrent Neural Network architecture for tumor cell detection,which gave accuracy of about $90 \%$.

\section{METHODOLOGY}

World Health Organization (WHO) has graded brain tumors , into grade 1 and 2 tumors that are low-grade tumors known as benign tumors, or grade 3 and 4 tumors which are high-grade tumors also called as malignant tumors, A Malignant tumor is harmful and life-threatening. Benign brain tumors do not contain cancer cells and grow gradually. They do not spread and usually stay in one region of the brain, whereas malignant brain tumors contain cancerous cells and grow quickly and spread to other brain and spine regions as well as according to brain health behaviour.

The flow chart in Fig. 3 describes the various steps involved in the process. The process involves data acquisition, preprocessing, segmentation of the brain MRI image and Classification of Brain MRI Images. Each of the steps are explained in detail below.

\section{A Image Acquisition}

It is the first step in the proposed methodology, in which this stage of operations are done using the suitable hardware such as mobile phone, digital camera and many other devices for the collection of the data source from unified surface. The image acquisition is the initial step for every image processing system, by the acquisition of the image various available techniques are performed according to the specific need. For the process of image acquisition in particular to the MRI data an high precision devices are deployed for the capturing of the brain data [4]. 


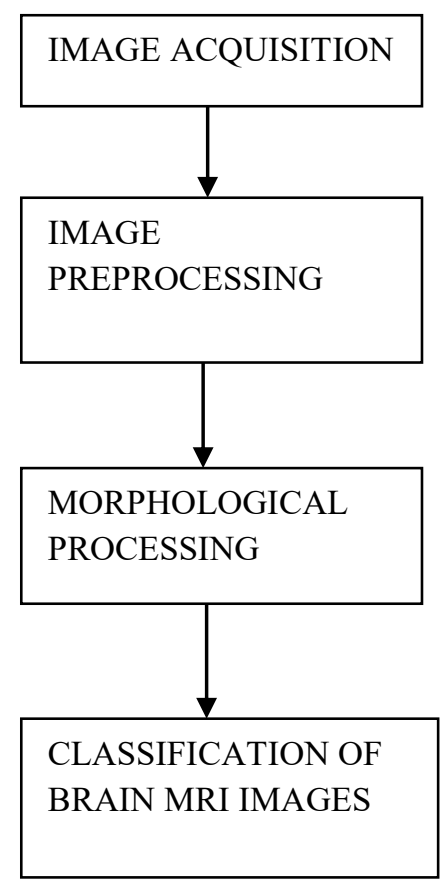

Figure 3. Proposed Methodology

\section{Image Pre processing}

This step is next process after image acquisition, MRI data is degraded by several noises like speckle, Gaussian, and Salt and Pepper noise. Through denoising the corrupted images can be converted to high quality images can be obtained. Normally the noise present in the signal is removed by the conventional methods used in the machine. The periodic noise existence will substantially reduce the effectiveness in conclusion and involves a great level of strength in the analysis of the MRI images. Each method used in denoising the MRI has its own challenges and advantages. Various approaches have been established for eliminating noises based on frequency spectrum and statistical property distribution. At all stages of the image acquisition unit, noise is introduced. The noises are Gaussian or non-Gaussian which are introduced in MRI due to several reasons. The signal and the noise are statistically independent of each other. Removal of noise from an MRI image is the one of the vital responsibilities in the MRI recognition system [15].

\section{Types of noises}

\section{i) Salt and pepper noise (Impulse noise)}

The impulse noise which are also known salt and pepper noise, as is caused by the malfunctioning and fault memory locations and in the channel used for transmission. For MRI images degraded by salt-and-pepper noise, the noisy pixels are found out by a minimum or maximum values randomly. A dataset with salt-pepper noise is taking either white pixel in dark region or black pixel in white region represented as value 1 and 0 respectively. From the existing systems it can be listed that, Compared with other linear techniques, median filter is used in the basic image processing system to remove salt-and-pepper noise because of its less sensitiveness as the denoising power and computational efficiency, the median filter is better and its popular nonlinear filter for removing impulse noise without affecting the sharpness of the image under filtration. [15].

\section{ii) Speckle noise}

Speckle noise is attributed to random interference between the coherent returns, it is a multiplicative noise and can be found in almost all coherent imaging systems such as SAR (Synthetic Aperture Radar) imagery, acoustics and laser Fully developed speckle noise has the characteristic of multiplicative noise [15].

\section{iii) Gaussian noise}

This kind of noise is a bell-shaped probability distribution function, Gaussian distributed noise value to the original pixel value. Although, theoretically speaking, the PDF is non-zero everywhere between $-\infty$ and $+\infty$, it is customary to consider the function 0 beyond $\mu \pm 3 \sigma$ [15]. Approximately $90 \%$ of the values are contained between $\mu \pm$ $2 \sigma$ and $70 \%$ of the values are contained between $\mu \pm \sigma$.

\section{Image quality (performance) metrics}

There exist different matrices to evaluate the temperament of the image processing strategies available. With the utilization of the measures available we were able to evaluate the raw image data and the processed image for assessment of the quality of it for the implication of the reference image with denoised image with proposed algorithm. In an conventional approach, both the images before and after processing need to be undistinguishable. In this present context SSIM, MSE and PSNR are used as image quality assessment but the parameters are not limited to it, other researches they may include FSIM and CoC.

\section{i) Peak signal to noise ratio (PSNR)}

PSNR gives the ratio between possible power of a signal and the power of corrupting noise present in the image as given in (1).[15]

$$
\operatorname{PSNR}=10 \log \left[\frac{L^{2}}{M S E}\right]
$$

where $\mathrm{L}$ is the dynamic range of the image, Ex. for 8-bit image, $\mathrm{L}=255$.

\section{ii) Mean squared error (MSE) or root mean squared error (RMSE)}

The root-mean-square deviation (RMSD) or rootmean-square error (RMSE) is a measure of differences between values predicted by a model and the values observed as given in (2). [15]

$\mathrm{MSE}=\frac{1}{M N} \sum_{i=1}^{M} \sum_{j=1}^{N}\left(I_{(i, j)}-J_{(i, j)}\right)^{2}(2)$

Where I and $\mathrm{J}$ are images of size $\mathrm{M} \mathrm{X} \mathrm{N}$.

\section{iii) Structural Similarity Index Measure (SSIM)}

The Structural SIMilarity (SSIM) index is a measue of the similarity between two images. The SSIM index is a quality measure of one of the images being compared, provided the other image is regarded as of perfect quality.

\section{Proposed denoising methodology}


The denoising techniques remove the noises as well as preserve the edges of an MRI. There are various kinds of filters available for removing the noise from the MRI and different filters have their own characteristic. The spatial domain filtering are further classified into linear and nonlinear filtering. Generally linear filtering techniques are used because of its speed of removing noise, but it does not preserve edges of images in efficient manner. On the other hand, nonlinear techniques preserve edges of images in a better way as compared to linear one. And, the non-linear filtering is used to remove an impulsive noise. The linear filtering method includes Gaussian filter, mean filter, poisson filter, Weiner filter.The non-linear filtering techniques include Median filter,Bilateral filter and Anisotropic diffusion filter.

\section{A. Image Segmentation}

Morphological operations are a set of operations where images are processed based on their shapes. A structuring element is applied to an input image and an output image is generated.

Erosion and dilation are most basic morphological operations. Erosion is used to diminish the features of an image and erodes away the boundaries of the foreground object. Dilation is used to accentuate features and increases the object area.

\section{B. Classification of Brain MRI Images}

The last step is the Classification of Brain MRI Images in Healthy brain and Brain with Tumor. Hybridized Convolutional Neural Network is used for this Classification.

\section{i) Architecture of CNN}

The combination of multiple linear and non-linear transformations of the input data forms deep learning architecture that are aimed at yielding more abstract and ultimately more useful representations. Since these methods have provided outstanding performance in different computer vision and pattern recognition tasks, there has been an increase in their popularity. These deep learning architectures have evolved from multilayer neural networks that involve different design and training strategies to make them competitive. These strategies incorporate spatial invariance, hierarchical feature learning and scalability. Another characteristic of this approach includes feature extracting that is a part of the learning process. This includes layers of deep learning architectures that help in finding an appropriate representation of input images in terms of multilevel visual feature maps that can be learnt.

In medical image processing, convolutional neural network is widely used to classify brain tumors. CNN contains advanced features which solves the problem with less time complexity. In this paper, a new CNN is suggested to classify whether the input MRI image has a tumor or not. Basically, CNN contains three important layers, such as a convolutional layer, max-pooling layer, and fully connected layer. The MRI image will be given as input to the first layer called a convolutional layer. Once the convolutional layer is given input, the output is produced by computing a crossproduct among the weights and regions. If the input image size is $64 \times 64$, the resultant output image is given to the maxpooling layer. The pooling layer reduces the output by a given factor from the convolutional layers. At last, the fully connected layer class scores computes the required numbers of output classes. The architecture of CNN, consisting of three important layers, is shown in Fig. 4.

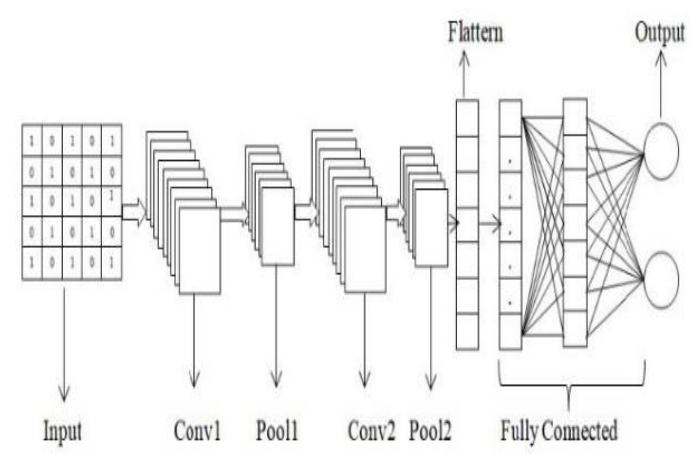

Figure 4. Architecture of the convolutional neural network

ii) Proposed Convolutional Neural Networks

The structure of the proposed network is depicted in Fig. 5. This network consists of fifteen layers consisting of five max-pooling layers, five convolutional layers, one flatten, two fully connected layers, and an output layer. By increasing the convolutional layer to neural network accuracy of classifier can be improved significantly. The noise in the input image is also reduced and the image is transformed that is more interpretable by the system. By increasing the pooling layers, features can be underlined and also size of the image will be reduced which in turn improves the training time.

The detailed description of each layer evolved in the design is given below.

\section{a) Input layer}

This is the primary layer, which is direct interface to fetch the MRI images from the user end for feature extraction at the next layer. 


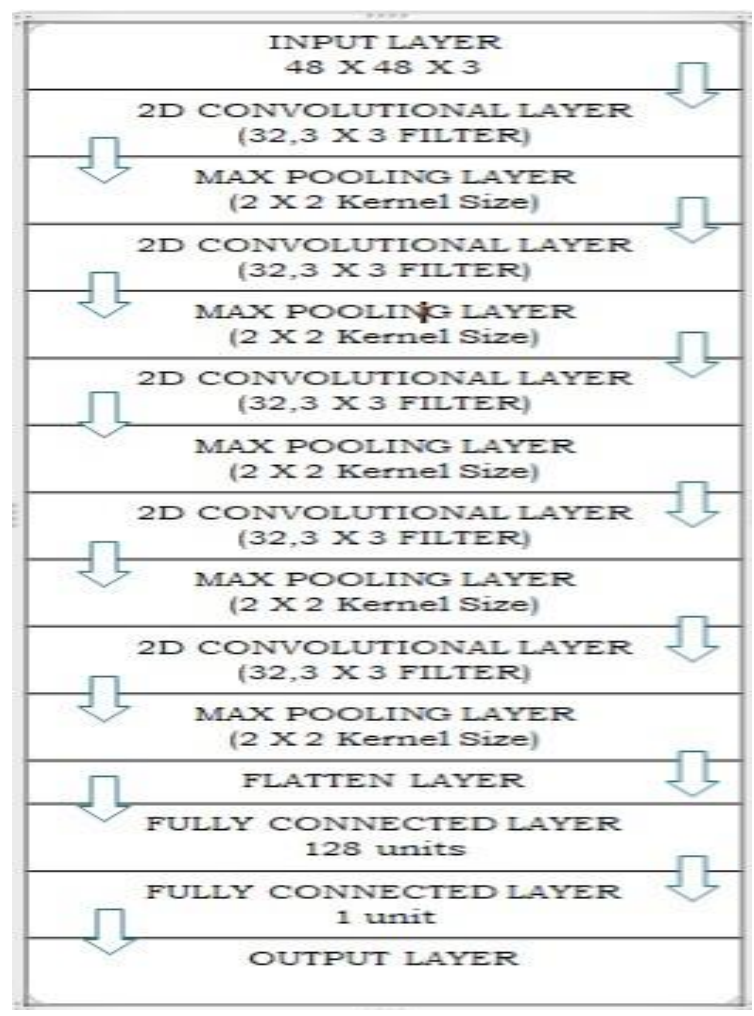

Figure 5. Proposed convolutional neural networks

\section{b) Convolutional layer}

The very next layer to the input layer is an bidimensional layer which is convolutional in nature. In this layer for the orientation of the required amount of filters are employed for the feature extraction from the input MRI image. With the extracted features from the MRI data are utilized for the calculation of the similarity indexes. The convolutional operation is conventionally termed as mutual product of $\mathrm{j}$ and $\mathrm{I}$ object functions with the interval $[0, \mathrm{k}]$ given by (3).

$$
[i * j](k)=\int_{0}^{\tau} \mathrm{f}(\tau) \mathrm{g}(\mathrm{k}-\tau) \mathrm{d} \tau
$$

The output image of the size $64 \times 64$ pixels with the performance of the convolution operation with $3 \times 3$ filters is applied on MRI image of dimension 64 X 64 X 3 , output image is represented in (4).

$$
\left[\frac{w i-f i+2 p a}{s t}\right]+1
$$

The output image with size of $64 \times 64$ is applied to the max-pooling layer. In the similar manner, all convolution layers are estimated in the proposed network.

\section{c) Re LU activation function}

This is a linear function, where the resultant obtained in accordance with the applied input if and only if it is non-negative. ReLU is a novel and mandatory triggering feature for various types of neural networks as a mannequin utilizes an simpler manner for the instruction to attain improved performance. Its operations are relatively linear for the values non zero, which implies the training of neural network in backward propagation. We applied the Rectified Linear Unit (ReLU) activation function in each convolutional layer. An Activation function converts the input weighted sum into that node's output. Rectifier Linear unit function is often used in hidden layers of the convolutional neural network. Mathematically, ReLU is represented by (5)

$$
f(z)=\max (0 ; z)
$$

Where $\mathrm{z}$ is the input when $\mathrm{z}$ is negative or equal to 0 , it transforms the negative input to 0 . When the input is greater than 0 , then the output will be 1 . So if the input is 0 then that neuron is a dead neuron in ReLU function and it won't be activated. The interesting feature of the ReLU function is the normalized values to zero for other intermediate values of non-positive hence it is nonlinear function for the rest of the values which will be given by (6).

$$
f(x)= \begin{cases}0, & x<0 \\ 1, & x \geq 0\end{cases}
$$

\section{d) Max pooling layer}

The pooling layer is very essential in terms of minimizing the dimension of operation. The minimization of the numbers of neurons in the output of the convolutional layer, the pooling algorithm is the hybrid combination of the adjacent members available in the output convolution matrix. Commonly used pooling algorithms are average and Max Pooling. In this article of research, the two dimensional convolution layer outcome is the feeding input to the maxpooling layer, the max-pooling layer output images can be estimated as shown in (7)

$$
\left[\frac{o+2 p a-2}{s t}\right]+1
$$

where, padding (pa) is 0 ,number of stride (st) is $2, \mathrm{O}$ is $64 \times 64$, and size of the filter (fi) is $3 \times 3$.So, the size of the image produced from the max pooling layer is $32 \times 32$ $([(64+0-2) / 2]+1)$. For the remaining max pooling layers, the same approach has been employed in the proposed architecture.

\section{e) Flatten layer}

With the following from the max-pooling and convolution operations and multi-dimensional tensor is mandatory at the output part, an uni dimensional tensor is required. This is attained in the flatten layer which are fully connected to the input layer.

\section{f) Fully connected layer}

Like feed-forward neural networks, each level's nodes are fully connected to all the nodes of the next layer in fully connected layer. For the classification function among the two different MRI images an activation function is much needed

In $\mathrm{CNN}$, the numbers of epochs are increased to adequately train the classifier which yields in better performance. Weights are being updated using weights updation function, while moving from epoch to epoch. It is shown in (8). 


$$
w_{i j}=w_{i j}+\Delta w_{i}
$$

where $\Delta w_{i j}=(l) E r r_{j} O_{i}$

\section{EXPERIMENTAL SETUP AND RESULT ANALYSIS}

\section{a) Image Preprocessing}

In the proposed work, an image with brain tumor has been taken. Figure 6 shows an image with brain tumor. Three types of noises are added to the original image. Fig. 7a) shows an image with salt and pepper noise. Fig. 8a) shows an image with Gaussian noise. Fig. 9a) shows an image with Speckle noise. Six types of filters namely Gaussian Filter, Poisson Filter, Bilateral Filter, Median Filter, Weiner Filter and Mean filter have been used to remove these noises. Fig. 7b), Fig. 8b) and Fig. 9b) shows Bilateral Filtered image. Peak Signal to Noise Ratio (PSNR) has been determined for all cases and is shown in Table 1.Table 2 shows comparison between different denoising methods by using image quality assessment parameters such as PSNR, MSE and SSIM.

Based on the results, it can be concluded that Anisotropic Diffusion Filter gives good results for image with all three types of noises.

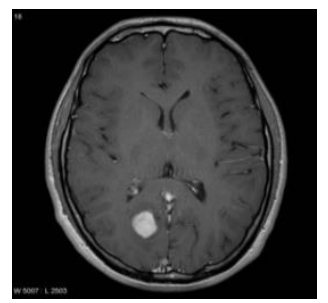

Figure 6. Original Image
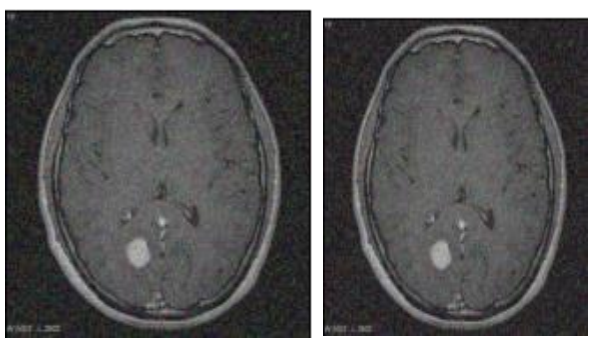

Figure 7a. Image with salt \& pepper noise Figure $7 b$. Bilateral Filtered Image

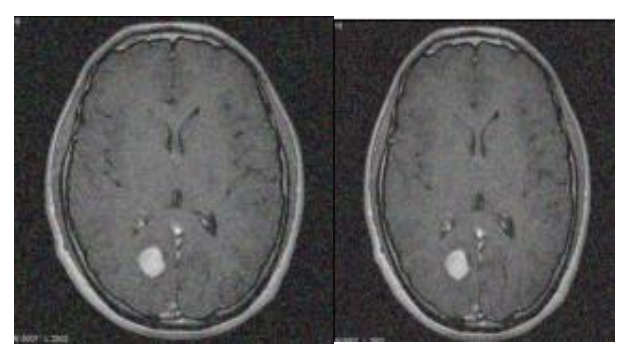

Figure 8a. Image with Gaussian noise Figure $8 b$. Bilateral Filtered Image

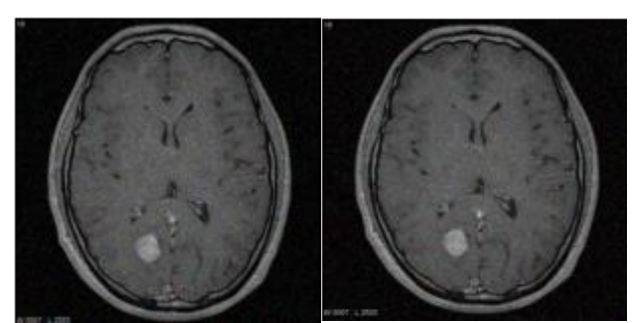

Figure 9a. Image with Speckle noise Figure 9b. Bilateral Filtered Image

Table 1. PSNR values for denoising salt and pepper, Gaussian and speckle noise.

\begin{tabular}{|l|l|l|l|l|}
\hline $\begin{array}{l}\text { Sl. } \\
\text { No. }\end{array}$ & $\begin{array}{l}\text { Image } \\
\text { Denoising } \\
\text { Methods }\end{array}$ & $\begin{array}{l}\text { PSNR } \\
\text { Salt \& } \\
\text { pepper } \\
\text { Noise, }\end{array}$ & $\begin{array}{l}\text { PSNR } \\
\text { Gaussian } \\
\text { Noise }\end{array}$ & $\begin{array}{l}\text { PSNR } \\
\text { Speckle } \\
\text { Noise }\end{array}$ \\
\hline 1 & $\begin{array}{l}\text { Gaussian } \\
\text { Filter }\end{array}$ & 14.50 & 14.95 & 19.48 \\
\hline 2 & $\begin{array}{l}\text { Poisson } \\
\text { Filter }\end{array}$ & 13.66 & 14.11 & 18.62 \\
\hline 3 & $\begin{array}{l}\text { Bilateral } \\
\text { Filter }\end{array}$ & 23.29 & 23.92 & 25.67 \\
\hline 4 & $\begin{array}{l}\text { Median } \\
\text { Filter }\end{array}$ & 12.75 & 12.99 & 18.34 \\
\hline 5 & $\begin{array}{l}\text { Weiner } \\
\text { Filter }\end{array}$ & 21.58 & 22.09 & 26.56 \\
\hline 6 & $\begin{array}{l}\text { Mean } \\
\text { Filter }\end{array}$ & 13.62 & 14.08 & 18.69 \\
\hline 7 & $\begin{array}{l}\text { Anisotropi } \\
\text { c Diffusion } \\
\text { Filter }\end{array}$ & $\mathbf{2 2 . 5 0}$ & $\mathbf{2 3 . 0 0}$ & $\mathbf{2 7 . 4 5}$ \\
\hline
\end{tabular}

Table 2. Quantitative Analysis

\begin{tabular}{|l|l|l|l|l|}
\hline \multirow{2}{*}{$\begin{array}{l}\text { Sl. } \\
\text { No. }\end{array}$} & Image & $\begin{array}{l}\text { Denoising } \\
\text { Methods }\end{array}$ & \multicolumn{2}{|l|}{$\begin{array}{l}\text { Image Quality Assessment } \\
\text { Parameters }\end{array}$} \\
\cline { 3 - 5 } & PSNR & MSE & SSIM \\
\hline 1 & Gaussian Filter & 14.95 & 2028.71 & 0.29 \\
\hline 2 & Poisson Filter & 14.11 & 2467.20 & 0.12 \\
\hline 3 & Bilateral Filter & 23.92 & 76.54 & 0.98 \\
\hline 4 & Median Filter & 12.99 & 3034.13 & 0.18 \\
\hline 5 & Weiner Filter & 22.09 & 21.27 & 1.00 \\
\hline 6 & Mean Filter & 14.08 & 2286.42 & 0.24 \\
\hline 7 & $\begin{array}{l}\text { Anisotropic } \\
\text { Diffusion } \\
\text { Filter }\end{array}$ & $\mathbf{2 3 . 0 0}$ & $\mathbf{5 7 . 5 1}$ & $\mathbf{0 . 9 9}$ \\
\hline
\end{tabular}

\section{b) Image Segmentation}

Morphological operations were used to mark the edges accurately and the Brain portion was cropped from the entire MRI image as shown in Fig. 10.

First, image thresholding is done, and then a series of erosions and dilations is performed to remove any small regions of noise.

Second,Contours is found in the thresholded image and largest one is considered.

Third,the extreme points are marked.

At last the image is cropped. 

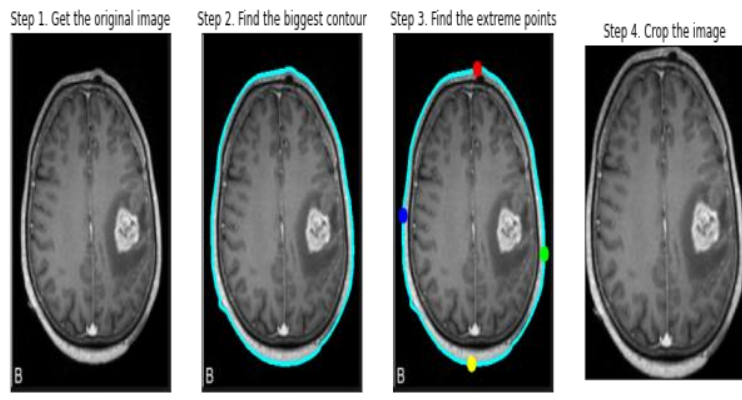

Figure 10. Results of Morphological Operations

\section{c) Classification of Brain MRI images.}

In this research article for the testing of the designed architecture of classifier for the brain tumour over the wide range of public available database it is chosen to operate with the KAGGLE dataset, in which 253 test images were united from BRATS database which is the association of 155 MRI images with tumor disorder and healthy person MRI images of 98 . In the context of the research proposed for the classifier approach it is considered with the 104 MRI images with disorder oriented dataset and 65 normal dataset of MRI images. For the classifier oriented investigation it is done with the 33 usual healthy person MRI data along with the 51 set of MRI images with few abnormalities and the distribution table of KAGGLE database is as shown in Table 3.

Table 3. KAGGLE Dataset Distribution.

\begin{tabular}{|l|l|l|l|}
\hline KAGGLE & $\begin{array}{l}\text { MRI } \\
\text { Images } \\
\text { with Tumor }\end{array}$ & $\begin{array}{l}\text { Healthy } \\
\text { MRI } \\
\text { Images }\end{array}$ & Total \\
\hline Training & 104 & 65 & 169 \\
\hline Testing & 51 & 33 & 84 \\
\hline Total & 155 & 98 & 253 \\
\hline
\end{tabular}

Some of the overall performance metrics considered is accuracy, confusion matrix, precision, recall, and F1 rating. From the matrix of confusion, most performance measurements are calculated. Performance evaluation metrics of any classifier can be calculated using four basic building blocks. Those are TP, FP, TN and FN. Those building blocks are explained in Table 4 in detail. Predicted values are taken on the $\mathrm{X}$-axis and actual values are taken on the $\mathrm{Y}$-axis.

Table 4. Building blocks of classifier in Confusion matrix

\begin{tabular}{|l|l|l|l|l|}
\hline \multicolumn{2}{|l|}{ CLASS } & \multicolumn{2}{|l|}{ PREDICTED } & \multirow{2}{*}{ Total } \\
\cline { 3 - 4 } \multicolumn{2}{|c|}{ Actual } & Positive & Negative & \\
\cline { 3 - 5 } & Positive & TP & FN & P \\
\hline \multicolumn{2}{|c|}{ Togative } & FP & TN & N \\
\hline \multicolumn{2}{|c|}{} & P & N & P+N \\
\hline
\end{tabular}

The rate correct identification and incorrect identifications can be categorized as two major segmentations logically viz., True and False respectively. As an sub set to make sure as similar to the confusion matrix for grouping it can be broadly fall into Positive and Negative values making it into four various corners of the confusion matrix such as TP,TN,FP \& FN termed as True
Positive, True Negative, False Positive and False Negative respectively

Performance of TP rate has been estimated by Sensitivity, it computes the percentage of positive cases that are correctly classified from the actual positive cases of dataset using (10).

$$
\text { Sensitivity }=\frac{(T P)}{(T P+F N)}
$$

Precision is the parameter used for the measurement of the exact correct prediction which can be given by (11). it computes the percentage of positive cases that are correctly.

$$
\text { Precision }=\frac{T P}{F P+T P}
$$

The classifier performance is gauged in terms of percentage for all the cases such as positive and negative in terms of accuracy which can be given by (12).

$$
\text { Accuracy }=\frac{(T N+T P)}{(T N+T P+F N+F P)}
$$

For the accurate measurement of the recalls and precisions in an single attempt it is employed with calculation of the F1 Score which can be interpreted as shown in (13)

$$
\text { F1score }(F)=\frac{2 X \text { Precision } X \text { Recall }}{\text { Precision }+ \text { Recall }}
$$

Performance of true negative rate has been estimated by Specificity, it computes the percentage of negative cases that are correctly classified from the actual negative cases of dataset using (14).

$$
\text { Specificity }=\frac{T N}{F P+T N}
$$

Experimentation was performed in Python (Google Colab Notebook) with the downloaded KAGGLE dataset. The resultant counts returned by the building blocks of classifier are given in Fig. 11. 


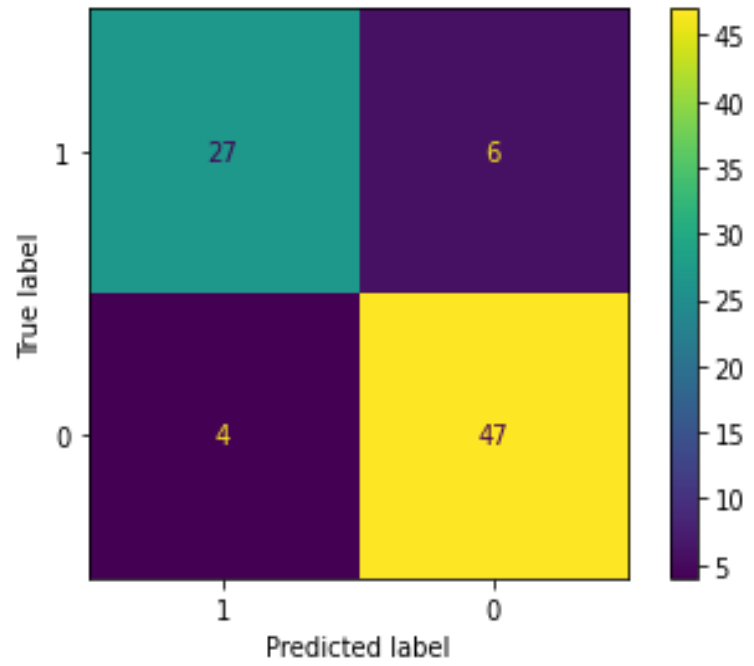

Figure 11. Contingency matrix for the proposed model

Obtained result interpretation is carried out with the confusion matrix, upon the test procedure of 84 different variants of data images with the $\mathrm{CNN}$ classifier utilized for training of the 169 data images, in which these are classified as true positives as 27 cases with true negative as 47 classification out of 84 data sets, the remaining 10 data sets has resulted as $40 \%$ of false positive and $60 \%$ as false negatives. The overall designed model has reached F1 score of $90 \%, 89 \%$ of precision, $92.15 \%$ of specificity and accuracy as $88.81 \%$ as tabulated in Table 5 and represented in Fig. 12.

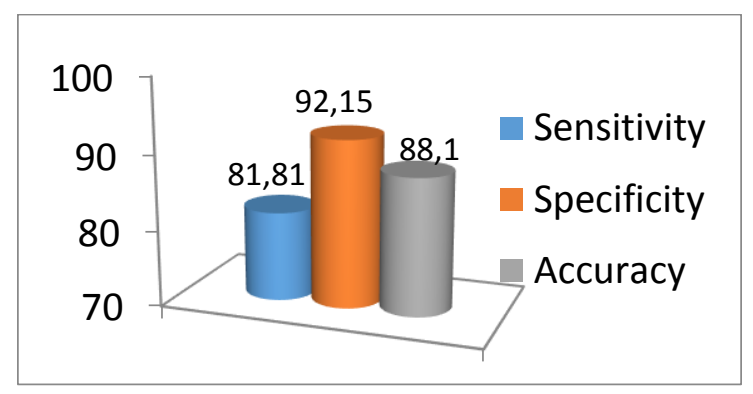

Figure 12. Performance Analysis of the proposed model

\begin{tabular}{|l|l|l|l|l|}
\multicolumn{5}{c|}{ Table 5. Performance criteria } \\
\hline & $\begin{array}{l}\text { Preci } \\
\text { sion }\end{array}$ & Recall & $\begin{array}{l}\text { F1- } \\
\text { score }\end{array}$ & Support \\
\hline NO & 0.87 & 0.82 & 0.84 & 33 \\
\hline YES & 0.89 & 0.92 & 0.90 & 51 \\
\hline Accuracy & \multicolumn{3}{|c|}{0.88} & 84 \\
\hline
\end{tabular}

\section{d) Performance analysis of Proposed Model}

For the pictorial representation of the accuracy and training data loss rate and testing data with fixed 35 number of epoch is done in Fig. 13 (a) and (b). When the epoch number is increased to 50 which is represented in Fig. 14 (a) and (b). With the comparative analysis from Fig. 13 and 14, it can be clearly stated that accuracy is improved with the increased number of epochs, it should not be over sighted the effect of training and testing data on accuracy also linearly increases.
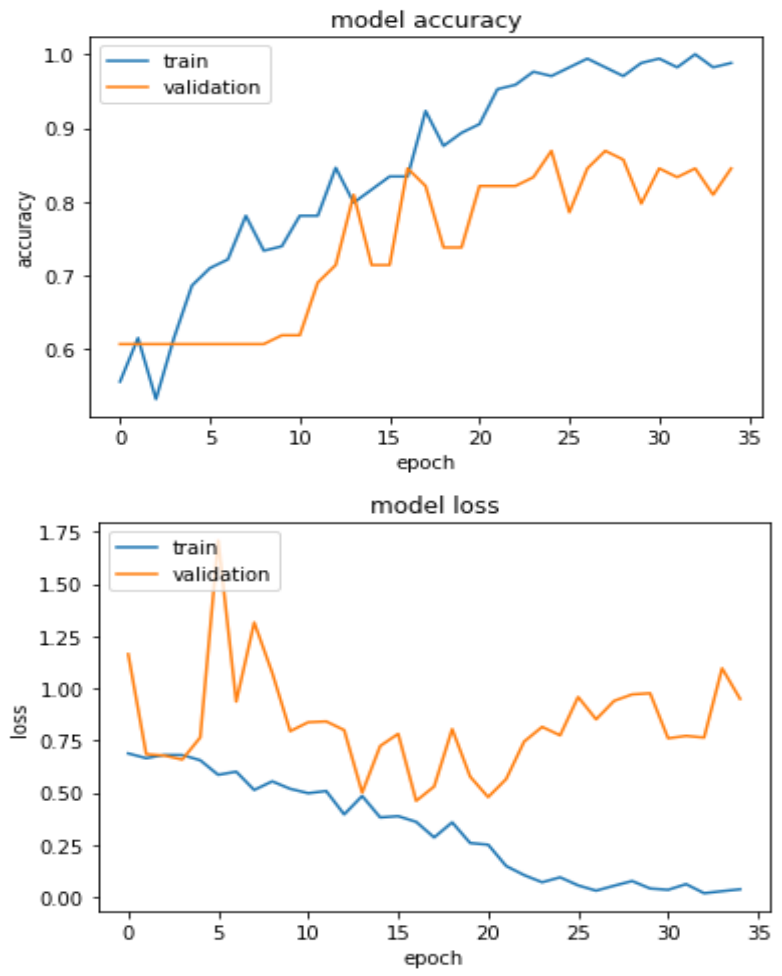

Figure 13 (a). Accuracy of proposed method with epoch $=$ 35; (b). Loss of proposed method epoch $=35$
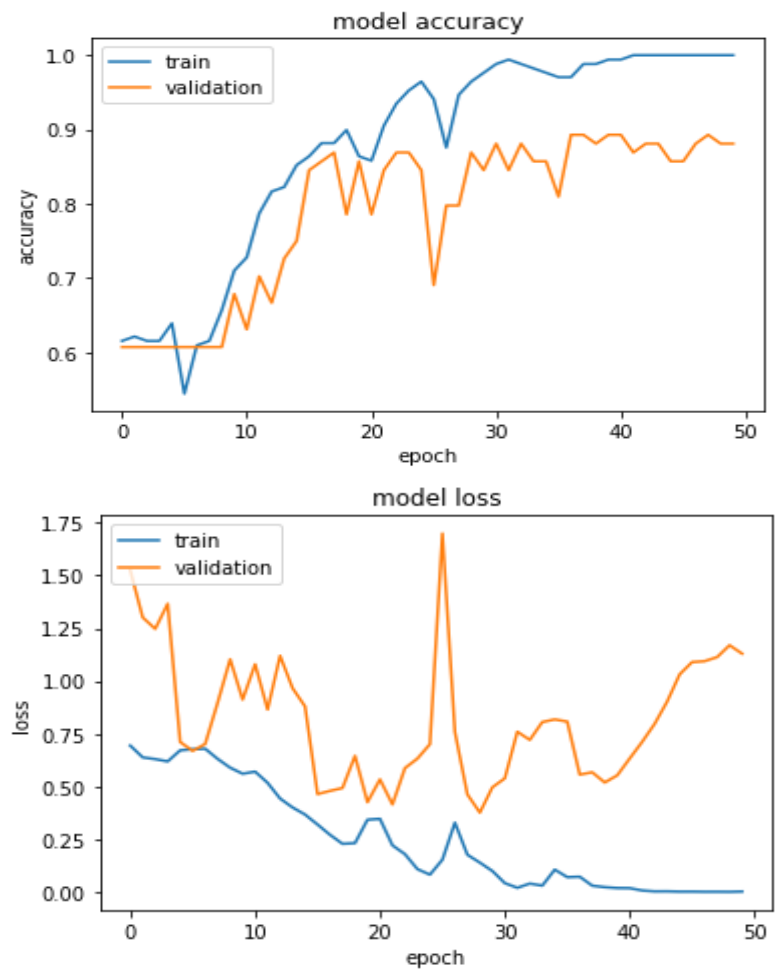

Figure 14 (a). Accuracy of proposed method with epoch $=$ $50 ;$ (b). Loss of proposed method epoch $=50$

\section{v. CONCLUSION}

In the present research context, it is considered with KAGGLE dataset for the analysis of the classifier 
performance. Initially, MRI image denoising was done using various filters. It was proved that Anisotropic diffusion filter can be used for all three types of noises. Morphological Operations can be done to segment brain from MRI image, in the training phase for CNN classifier, it achieved with the almost one hundred normal images and one hundred and fifty images with the abnormality in total more than two hundred and fifty images were considered for the data model. The designed Convolutional Neural Networks were aimed to identify brain tumor from the database under consideration with the classifier approach is carried out with 51 image sets with abnormalities and 33 normal images making in total of 84 image sets. A hybrid CNN model has been presented, with feedforward neural network as a seed, with the incremental values of epochs, it is assured to have higher accuracy due to higher rate of training states. An individual Epoch is defined as the entire process to reach the output layer from the input layer for the calculation of the Accuracy, Precision, Specificity, Sensitivity and F1 score. It is observed from the results that $85 \%$ of accuracy for the CNN with 35 number of epoch and it is attained with $88 \%$ of accuracy when the linear enhancement of the epochs. The major gain in the classifier proposed to the MRI images of the brain was more accurate when compared with the less number of epochs. Other factor of improvement as an future scope is to minimize the processing time to process the tumor images as an design constraint and also to increase the accuracy.

\section{References}

[1] NBTS, National Brain Tumor Society: Quick brain tumor facts, 2021. Available from:https://braintumor.org/brain-tumorinformation/brain-tumor-facts/.

[2] Badza, M.M. Barjaktarovic, M.C,"Classification of Brain Tumors from MRI Images Using a Convolutional Neural Network". Appl.Sci,MDPI, Basel, Switzerland, March 2020.

[3]T. Ruba, R. Tamil selvi, M. ParisaBehamand,N.Aparna,"Accurate Classification and Detection of Brain Cancer Cells in MRI and CT Images using Nano Contrast Agents",Biomedical \& Pharmacology Journal, Vol. 13(3), p. 12271237,September 2020.

[4]K.Kalaiselvi, C. Karthikeyan, M. Shenbaga Devi, C. Kalpana, "Improved Classification of Brain Tumor in MR Images using RNN Classification Framework", International Journal of Innovative Technology and Exploring Engineering (IJITEE) ISSN: 2278-3075, Volume-9 Issue-3, 1098-1101, January 2020

[5] Gokalp Cinarer, Bülent Gursel Emiroglu, Recep Sinan Arslan, Ahmet Haşim Yurttakal, "Brain Tumor Classification Using Deep Neural Network", Advances in Science, Technology and Engineering Systems Journal Vol. 5, No. 5, 765-769, October 2020.

[6] Venkata Ramakrishna Sajja, Hemantha Kumar Kalluri,"Classification of Brain Tumors Using Convolutional Neural Network over Various SVM Methods" International Information and Engineering Technology Association, Vol.25,No.4,489-495,August 2020 .
[7]Muhammad Attique Khan, et.al. "Multimodal Brain Tumor Classification Using DeepLearning and Robust Feature Selection: A MachineLearning Application for Radiologists", Diagnostics,MDPI, Basel, Switzerland, August 2020.

[8] Hassan Ali Khan, Wu Jue, Muhammad Mushtaq and Muhammad Umer Mushtaq.'Brain tumor classification in MRI image using convolutional neural network", Mathematical Biosciences and Engineering, Volume 17, Issue 5, 6203-6216, September 2020.

[9] Dr.R.C.Suganthe, G.Revathi, S.Monisha, R.Pavithran, "Deep Learning Based Brain Tumor Classification Using Magnetic Resonance Imaging",Journal of Critical Reviews,ISSN- 2394-5125,Vol 7, Issue 9,May 2020.

[10] Ali Mohammad Alqudah, Hiam Alquraan, Isam Abu Qasmieh, Amin Alqudah, Wafaa Al-Sharu, "Brain Tumor Classification Using Deep Learning Technique - A Comparison between Cropped, Uncropped, and Segmented Lesion Images with Different Sizes", International Journal of Advanced Trends in Computer Science and Engineering, 8(6), ISSN 22783091,December 2019, 3684-3691.

[11] Salim Ouchtati, et.al.,'Novel Method for Brain Tumor Classification Based on Use of Image Entropy and Seven Hu's Invariant Moments", Traitement du Signal, Lavoisier,36 (6), pp.483-491, 2019.

[12] Shaik Basheera, and M. Satya Sai Ram, "Classification of Brain Tumors Using Deep Features Extracted Using CNN “, International Conference on Applied Physics, Power and Material Science, Conf. Series 1172, 2019.

[13] Miss Krishna Pathak, et al., "Classification of Brain Tumor Using Convolutional Neural Network", Proceedings of the Third International Conference on Electronics Communication and Aerospace Technology [ICECA 2019],IEEE Conference Record \# 45616; IEEE Xplore ISBN: 978-1-7281-0167-5,2019.

[14] S. Deepak, P.M. Ameer., "Brain tumor classification using deep CNN features via transfer learning", Computers in Biology and Medicine 111, Elsevier Ltd, 2019.

[15] Ravikumar Gurusamy and Dr.Vijayan Subramaniam, "A Machine Learning Approach for MRI Brain Tumor Classification”, CMC, vol.53, no.2, pp.91-108, 2017.

[16] R.Anjali, S.Priya," An Efficient Classifier For BrainTumor Classification", International Journal of Computer Science and Mobile Computing, Vol.6 Issue.8, August- 2017, pg. 40-48.

[17]Dipali M. Joshi,et.al., "Classification of Brain Cancer Using Artificial Neural Network",2nd International Conference on Electronic Computer TechnologyIEEE,2010.

[18] Suresha D, N Jagadisha, H S Shrisha, K S Kaushik, "Detection of Brain Tumor Using Image Processing", Fourth International Conference on Computing Methodologies and Communication, IEEE Xplore ,2020.

[19] Shreyasi Ghose,Nishi Singh,Prabhishek Singh, "Image Denoising using Deep Learning:Convolutional Neural Network", IEEEXplore, 2020.

[20] Anandbabu Gopatoti, Merajothu Chandra Naik, Kiran Kumar Gopathoti,"Convolutional Neural Network Based Image Denoising for Better Quality of Images", 
International Journal of Engineering \& Technology, 7 (3.27),pp356-361,2018.

[21] Pattabiraman Ventakasubbu and Parvathi Ramasubramanian,'Deep Learning-based Brain Tumour Segmentation", IETE Journal of Research, DOI: 10.1080/03772063.2021.1919219.

[22] Rafeek Mamdouh, Hazem M. El-Bakry, Alaa Riad, Nashaat El-Khamisy," Converting 2D-Medical Image Files "DICOM" into 3D- Models, based on Image Processing, and Analysing Their Results with Python Programming", WSEAS Transactions on Computers, DOI: $10.37394 / 23205.2020 .19 .2$.

Creative Commons Attribution License 4.0 (Attribution 4.0 International, CC BY 4.0)

This article is published under the terms of the Creative Commons Attribution License 4.0

https://creativecommons.org/licenses/by/4.0/deed.en_US 